Marco Bertoni, Danilo Cavapozzi, Martina Celidoni and Elisabetta Trevisan

\title{
4 Assessing the material deprivation of older Europeans
}

- An aggregate measure of material deprivation is defined by summarising in a single index the failures in the affordability of basic needs and the experience of financial difficulties

- Aggregation is carried out under alternative approaches

- Our results consistently show that material deprivation of older Europeans is lowest in Scandinavian countries and highest in Southern Europe, Slovenia and Estonia

\subsection{How to aggregate different aspects of material deprivation}

A growing literature in recent years has focused on the assessment and analysis of material deprivation as a measure of material conditions. Material deprivation is usually defined as the extent of functioning failures in the dimension of material well-being. Material deprivation is expressed as households' inability to afford desired goods or services because of lack of economic resources. It is often considered a pathway to social exclusion.

Several contributions assess deprivation by considering the functioning failures of households with respect to a battery of items (see Bellani 2013 or Bossert et al. 2013). All these studies rely on the recognition that deprivation is a multifaceted state that can be properly described by taking into account several aspects of individuals' quality of life, including but not limited to financial outcomes.

Our chapter contributes to this literature by exploiting new information collected in the fifth wave of SHARE to provide a snapshot of the material deprivation among older people in Europe at the time the countries continue to struggle with the consequences of the economic crisis. Using data from SHARE Wave 2, Angelini et al. (2009) show that even before the economic crisis a substantial proportion of older European individuals experienced financial difficulties. In this chapter we extend the approach to the measurement of material conditions and document the level of deprivation across Europe and its variation among SHARE countries after the crisis.

Aggregating household functioning failures across a number of deprivation items into a single index requires a weighting scheme that sets the relevance of 
each item in the index computation. It has been demonstrated that alternative weighting schemes can affect the predictions of the analysis (see Cavapozzi et al. forthcoming) and generate a different pattern of deprivation across different countries and groups of the population. For this reason, we examine three alternative weighting approaches to test the sensitivity of the derived indices to the choice of weights. This allows us to recommend a weighting approach that is used in the following chapters with respect to material and social deprivation. The three weighting schemes we use in this chapter are equal, hedonic and stated preferences. First, we adopt equal weighting by assigning to each item the same weight. Second, we derive a set of hedonic weights that assigns to each item a weight proportional to its correlation with life satisfaction. Third, we take advantage of the design of the SHARE questionnaire, which makes it possible to match each item considered in our exercise with the assessment about its importance to attain decent living conditions provided by the Eurobarometer survey. We draw data from Eurobarometer to derive a set of stated preference weights according to which each item will receive a weight proportional to the percentage of individuals who consider it as absolutely necessary to attain a decent standard of living.

We will make use of the so-defined three aggregate indices to analyse crosscountry differentials in the material deprivation of older Europeans and to assess to what extent the results of our analysis are sensible to the weighting scheme adopted.

\subsection{Material deprivation items}

Our material deprivation assessment is based on a set of eleven items that refer to two broad domains: failure in the affordability of basic needs and financial difficulties.

The failures with respect to each item are defined according to binary indicators that allow classifying households as deprived or not deprived. If households fail to reach a minimum target with respect to a given item, they are classified as "deprived" with respect to that item, otherwise they are classified as "not deprived". Our analysis is based on a sample consisting of almost 40,000 households.

As for basic needs, we look at the households' failures in the affordability of a minimal quantity of meat, fish, chicken, fruits and vegetables in their diet (at most twice a week) and in the affordability of heating costs to avoid feeling cold at home, the replacement of worn out clothes and shoes, the purchase of new needed glasses, visits to the dentist and visits to the doctor (MDI: meat, MDI: fruit, MDI: heating, MDI: clothing, MDI: shoes, MDI: glasses, MDI: dentist, MDI: doctor). For a full list of items and their description see Table 2.1 in chapter 2 in this volume). 
Figure 4.1 shows the number of deprivations in this domain by country. We notice that the proportion of households who do not experience deprivation is lowest in Estonia (less than 40\%). With the exception of Spain, Italy, Estonia and Slovenia, at least 60 per cent of households in all countries do not experience any deprivation with respect to the affordability of basic needs. Denmark, Sweden and Switzerland are the countries that combine the highest proportion of not deprived households (about 90\%) with a fairly low presence of households deprived of four or more items. The highest level of deprivation with respect to four or more items is found in Estonia and Italy.

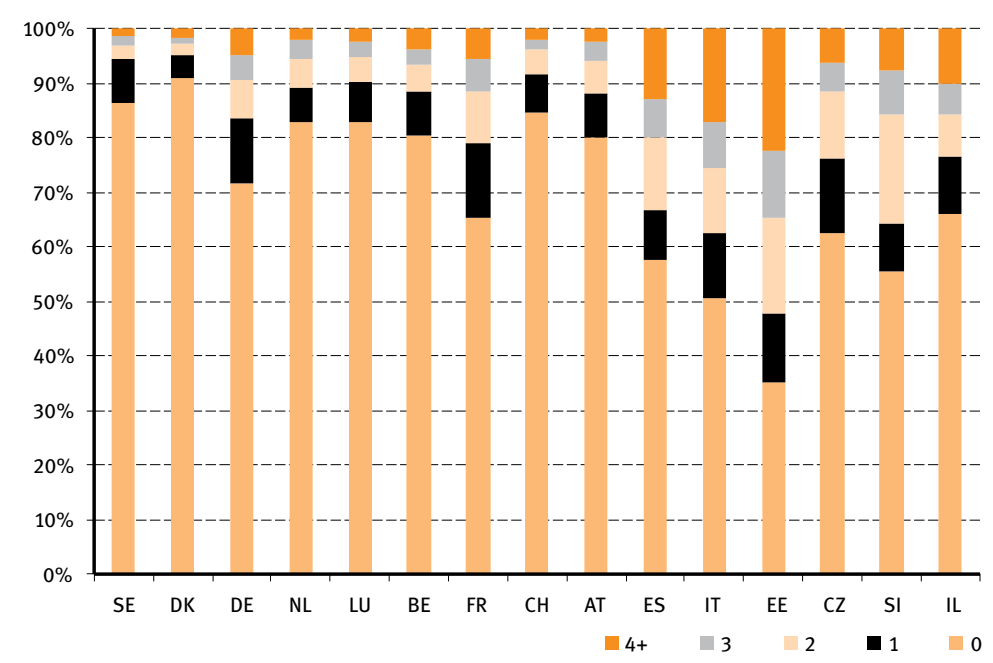

Figure 4.1: Number of deprivation in basic needs

Notes: $\mathrm{n}=39,574$. Calibrated cross-sectional household weights are used

Source: SHARE Wave 5 release 0

Within the financial difficulties domain, we consider the following three items. The first item refers to being in arrears with the payment of rents, the repayment of mortgages or loans on dwelling or having overdue bills (MDI: arrears). The second and the third items refer to failures in the affordability of a week long holiday away from home once a year and the affordability of paying an unexpected expense without borrowing any money (MDI: holiday, MDI: expense). Figure 4.2 shows the distribution of the number of deprivation in this domain. While Estonia is again the country with the lowest number of not deprived households (slightly more than $20 \%$ ), this proportion is highest for Scandinavian countries, the Netherlands, Luxembourg and Switzerland. 


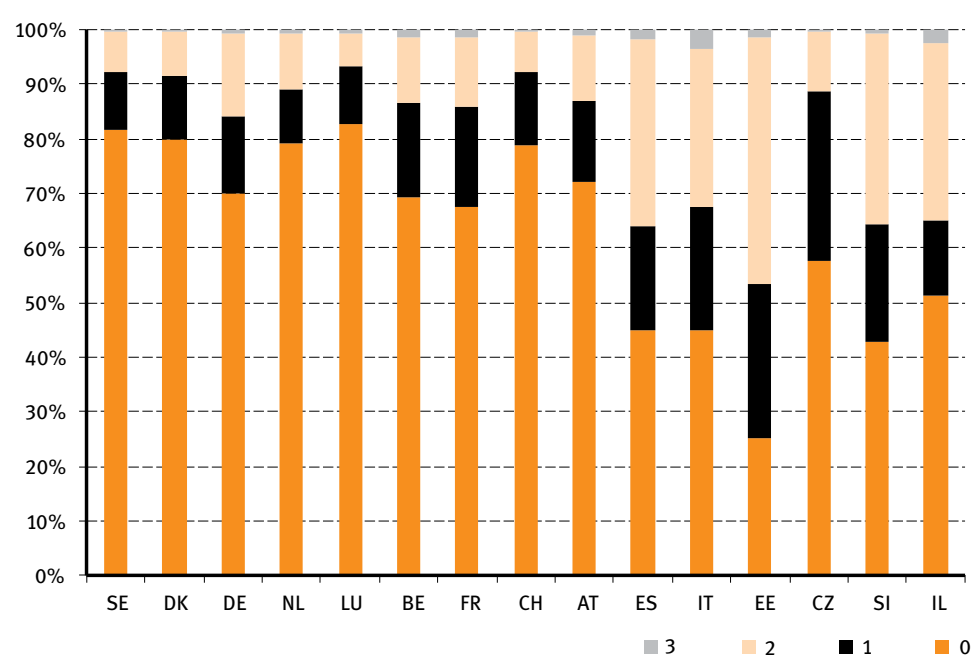

Figure 4.2: Number of deprivations in financial difficulties Notes: $n=39,574$. Calibrated cross-sectional household weights are used Source: SHARE Wave 5 release 0

\subsection{Alternative weighting schemes}

Our material deprivation index is computed as the weighted sum of the households' failures with respect to the set of the considered items. Following the classification discussed in Decancq and Lugo (2013), we will use three alternative weighting schemes: equal weighting, hedonic weighting and stated preference weighting.

Equal weighting depends on the value judgments of the analysts about the trade-offs between items. This scheme assigns the same weight to each item-specific indicator and implicitly assumes that all indicators are equally important for everyone.

Hedonic weighting reflects the correlation between the set of material deprivation items and self-assessed life satisfaction provided by respondents. More specifically, we derive hedonic weights by running an ordered probit regression of life satisfaction (measured on the scale from 0-10) on the set of material deprivation items considered and a full set of country dummies. The hedonic weight of each item is based on the corresponding estimated coefficient in the ordered probit equation.

Stated preference weights are based on the evaluations of a representative sample of individuals drawn from Eurobarometer data. In our analysis we use the Special Eurobarometer 279 on poverty and social exclusion conducted in 
2007, which asked respondents to determine the needs of the society in attaining decent living conditions. As in Bellani (2013) and Fusco et al. (2013), each item is assigned a weight with reference to the proportion of Eurobarometer respondents who deem being not deprived with respect to this item as absolutely necessary to attain a decent standard of living. Stated preference weights are calculated separately by country. In our exercise we restrict the Eurobarometer sample to individuals aged 50 or over living in the SHARE countries in order to preserve the comparability with the SHARE target population. Hedonic and stated preference weights have been standardised to sum up to one. Equal weights are standardised by definition. Stated preference weights are not available for Switzerland and Israel since these countries are not included in the Eurobarometer sample.

Figure 4.3 shows the weights attached to each item according to the three alternative weighting schemes. The figure clearly shows how the approach used to define weights affects the computation of the material deprivation index. For instance, the overall weight estimated for the financial difficulties domain is 39.90 per cent according to the hedonic weighting approach, it amounts to 27.27 per cent with equal weighting and it is just 20.97 per cent according to the stated preference weight based on Eurobarometer. This means that deprivation in all financial difficulties domain items will contribute less under the stated preference weighting scheme in defining the score of the material deprivation index. The reverse pattern is found if we look at the weights related to the affordability of new needed glasses, visits to the dentist or to the doctor. Whereas these items have an overall weight equal to about 40 per cent under the stated preference scheme, their weight according to the hedonic scheme approximately halves.

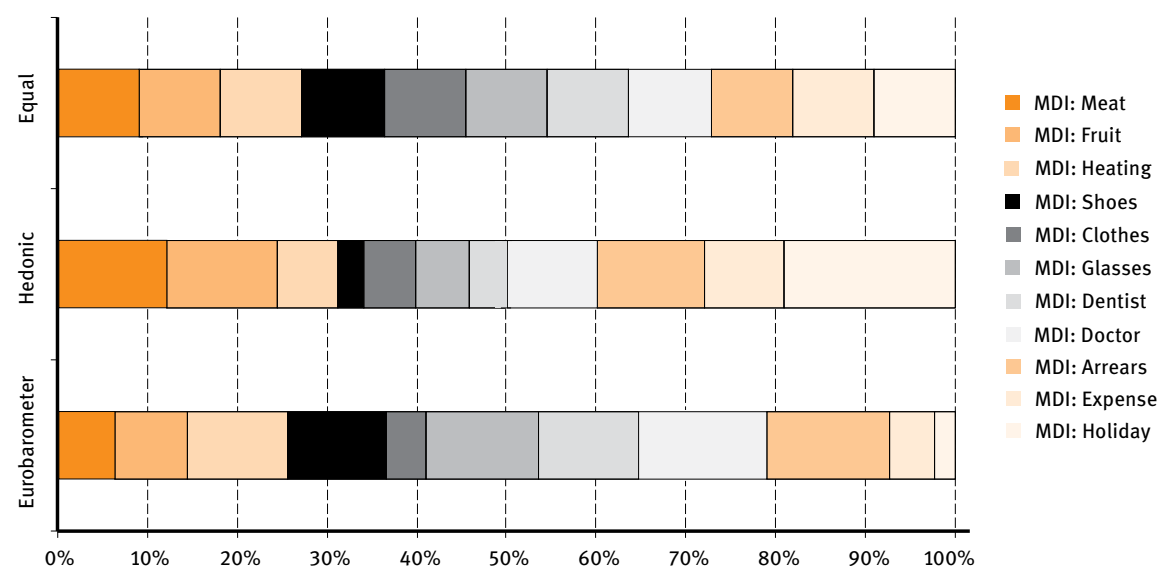

Figure 4.3: Weights assigned to specific deprivation items under different weighting schemes Source: SHARE Wave 5 release 0, Special Eurobarometer 279 


\subsection{Material deprivation score}

The previous sections of this chapter presented the key ingredients of our empirical exercise: items and weights. Our index of material deprivation is computed as the weighted sum of household failures with respect to the set of items considered. The index of material deprivation is a score that lies between 0 and 1 since deprivation in each item is described by dummy variables taking on value 1 when deprivation occurs and 0 otherwise and weights are standardised to sum up to one.

Figure 4.4 shows the country averages of the material deprivation scores according to the three weighting schemes considered. As expected, different weighting schemes are associated with different levels of material deprivation. On the one hand, for each country the material deprivation score based on equal and hedonic weighting do not differ significantly on average. On the other hand, stated preference weighting is always associated with lower averages of deprivation in all countries. This pattern might be due to the fact that, under stated preference weighting, items with a relatively lower prevalence of deprivation might be associated with higher weights and vice versa. As an example, deprivation with respect to the affordability of holidays has the highest prevalence (it is reported by $32 \%$ of the sample). The weight assigned to this item is high under the hedonic scheme but almost negligible under the stated preference scheme (see Figure 4.3). On the contrary, deprivation with respect to the affordability of new needed glasses has a much lower prevalence (10\%) but its relevance under the stated preference scheme is higher than in the hedonic case.

Despite the differences in the value of the weights applied, the overall ranking of the countries with respect to material deprivation is unaffected by the weighting scheme adopted. The only exception is the switch between France and the Czech Republic when moving between hedonic and stated preference weighting. This discrepancy appears to be minor, however, since the differences between the two countries are minimal even under hedonic weighting. Additionally, we carried out a formal Spearman rank correlation test, whose results support the hypothesis that the differences in the rankings emerging from the different weighting schemes considered are not statistically significant.

Regardless of the weighting scheme considered, Figure 4.4 shows the presence of a clear geographical gradient in material deprivation. Consistently with the evidence emerging when looking at the extent of deprivation by domain (Figures 4.1 and 4.2), Estonia and Italy are always the countries with the highest level of material deprivation, whereas Denmark and Sweden are those where material deprivation is lowest. The other Northern and Central European coun- 
tries experiment material deprivation levels more similar to those of the Scandinavian countries. On the other hand, Slovenia and Israel show levels of material deprivation close to those in Spain.

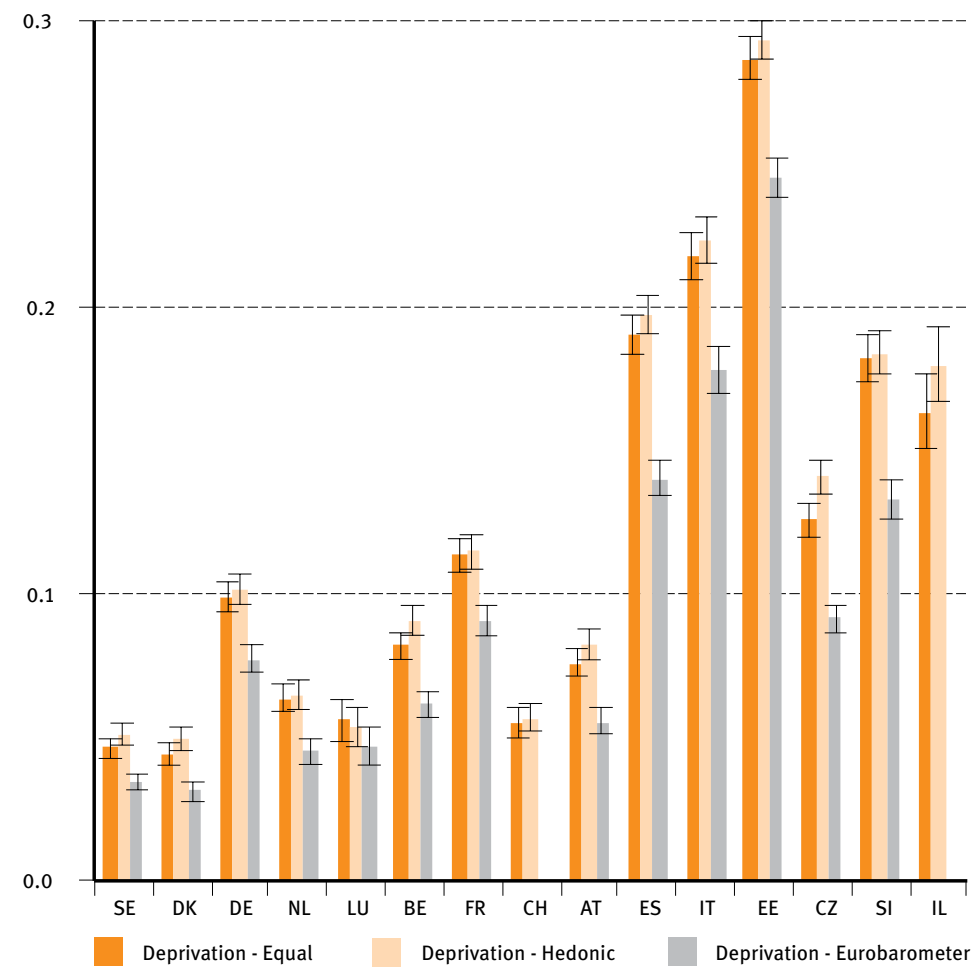

Figure 4.4: Material deprivation by country

Notes: $\mathrm{n}=39,574$. Calibrated cross-sectional household weights are used

Source: SHARE Wave 5 release 0

\subsection{Material deprivation in Europe}

We measured material deprivation of older individuals in Europe by developing aggregate measures of material conditions using a set of eleven items related to the affordability of basic needs and the experience of financial difficulties. The aggregation has been carried out using three alternative weighting schemes.

Our analysis documents the presence of a clear geographical gradient in material deprivation of older Europeans. Scandinavian countries are those experiencing the lowest levels of deprivation. Higher levels are found for Southern 
countries, Slovenia and Estonia. These rankings are confirmed regardless of the weighting scheme adopted.

Our findings suggest that in some countries the prevalence of material deprivation might be a pervasive reason of social exclusion among older individuals. Deprivation and exclusion are multifaceted conditions. Eradicating them requires coordinated policies intervening jointly on multiple aspects of older individuals' socioeconomic status. The framework used to derive the aggregate measures of material deprivation discussed in this chapter can serve as the basis of a proper evaluation of such policies.

Moreover, we demonstrated that in our exercise the country rankings of material deprivation are robust to the alternative methods of weighting the deprivation items. Given this conclusion, in the following chapter we derive a measure of material deprivation using an extended number of deprivation items and the hedonic approach to weighting. This approach on the one hand, is free from the need for additional evaluation of the importance of specific items (which is the case in stated preference weighting), and on the other, avoids assigning the same weights to items which may importantly differ with respect to their effect on welfare.

\section{References}

Angelini, Viola, Brugiavini, Agar, Weber, Guglielmo (2009): “Ageing and unused capacity in Europe: is there an early retirement trap?". In: Economic Policy 24, p. 463-508.

Bellani, Luna (2013): "Multidimensional indices of deprivation: the introduction of reference groups weights". In: Journal of Economic Inequality 11, p. 495-515.

Bossert, Walter, Chakravarty, Satya, D’Ambrosio, Conchita (2013): “Multidimensional poverty and material deprivation with discrete data". In: Review of Income and Wealth 59. No. 1, p. 29-43.

Cavapozzi, Danilo, Han, Wei, Miniaci, Raffaele: “Alternative weighting structures for multidimensional poverty assessment". Forthcoming in: Journal of Economic Inequality.

Decancq, Koen, Lugo, María Ana (2013): "Weights in multidimensional indices of well-being: an overview". In: Econometric Reviews 32. No. 1, p. 7-34.

Fusco, Alessio, Guio, Anne-Catherine, Marlier, Eric (2013): "Building a material deprivation index in a multinational context: lessons from the EU experience”. In: Berenger, Valerie, Bresson, Florent (Eds.): Poverty and social exclusion around the mediterranean sea.

New York: Springer Science+Business Media, p. 43-71. 